\title{
CPAS Preflight Drop Test Analysis Process
}

\author{
Megan E. Englert ${ }^{1}$ \\ UTC Aerospace Systems, Houston, TX, 77058 \\ and \\ Kristin J. Bledsoe ${ }^{2}$ \\ MRI Technologies, Houston, TX, 77058 \\ and \\ Leah M. Romero ${ }^{2}$ \\ Aerodyne, LLC., Houston, TX, 77058
}

Throughout the Capsule Parachute Assembly System (CPAS) drop test program, the CPAS Analysis Team has developed a simulation and analysis process to support drop test planning and execution. This process includes multiple phases focused on developing test simulations and communicating results to all groups involved in the drop test. CPAS Engineering Development Unit (EDU) series drop test planning begins with the development of a basic operational concept for each test. Trajectory simulation tools include the Flight Analysis and Simulation Tool (FAST) for single bodies, and the Automatic Dynamic Analysis of Mechanical Systems (ADAMS) simulation for the mated vehicle. Results are communicated to the team at the Test Configuration Review (TCR) and Test Readiness Review (TRR), as well as at Analysis Integrated Product Team (IPT) meetings in earlier and intermediate phases of the pre-test planning. The ability to plan and communicate efficiently with rapidly changing objectives and tight schedule constraints is a necessity for safe and successful drop tests.

\section{Nomenclature}

$\begin{array}{ll}\text { ACES } & =\text { Advanced Cockpit Environment Simulation } \\ \text { ADAMS } & =\text { Automatic Dynamic Analysis of Mechanical Systems } \\ \text { conops } & =\text { Concept of Operations } \\ \text { CPAS } & =\text { Capsule Parachute Assembly System } \\ \text { CPSS } & =\text { Cradle and Platform Separation System } \\ \text { EDU } & =\text { Engineering Development Unit } \\ \text { FAST } & =\text { Flight Analysis and Simulation Tool } \\ \text { IPT } & =\text { Integrated Product Team } \\ \text { JETS } & =\text { JSC Engineering, Technology, and Sciences (contract) } \\ \text { JSC } & =\text { Johnson Space Center } \\ \text { NASA } & =\text { National Aeronautics and Space Administraion } \\ \text { PTV } & =\text { Parachute Test Vehicle } \\ \text { TCR } & =\text { Test Configuration Review } \\ \text { TRR } & =\text { Test Readiness Review }\end{array}$

\footnotetext{
1 Analysis Engineer; GN\&C, Aerosciences, and Tech Transfer/EPO; 2224 Bay Area Blvd., Houston, TX. Nonmember.

${ }^{2}$ Analysis Engineer; GN\&C, Aerosciences, and Tech Transfer/EPO; 2224 Bay Area Blvd., Houston, TX. AIAA Member.
} 


\section{Introduction}

A preliminary test matrix that defines primary test objectives, test article, and type and number of parachutes to be used to meet those objectives, was developed prior to the beginning of the Engineering Development Unit (EDU) test series to provide initial direction for achieving EDU goals. The test matrix provides a starting point for the planning of each EDU test. It is generally considered to be fluid, changeable to meet the evolving needs and goals of the test program. Refinement of the test matrix is generally accomplished with input from the CPAS Management, Test Operations, Hardware, and Analysis Integrated Product Teams (IPT). These teams include representatives from NASA, Johnson Space Center (JSC) Engineering, Technology, and Science (JETS) contract, Airborne Systems, and Lockheed Martin.

The CPAS team has developed a process for planning and execution of each EDU drop test. The tests are complex and involve many teams and disciplines. This paper will discuss the process as it applies to the JETS Simulation and Analysis Team. This process includes five distinct phases that are tailored to address test-specific needs within schedule constraints. The top-level process flow is shown in Figure 1 and discussed in the following sections.

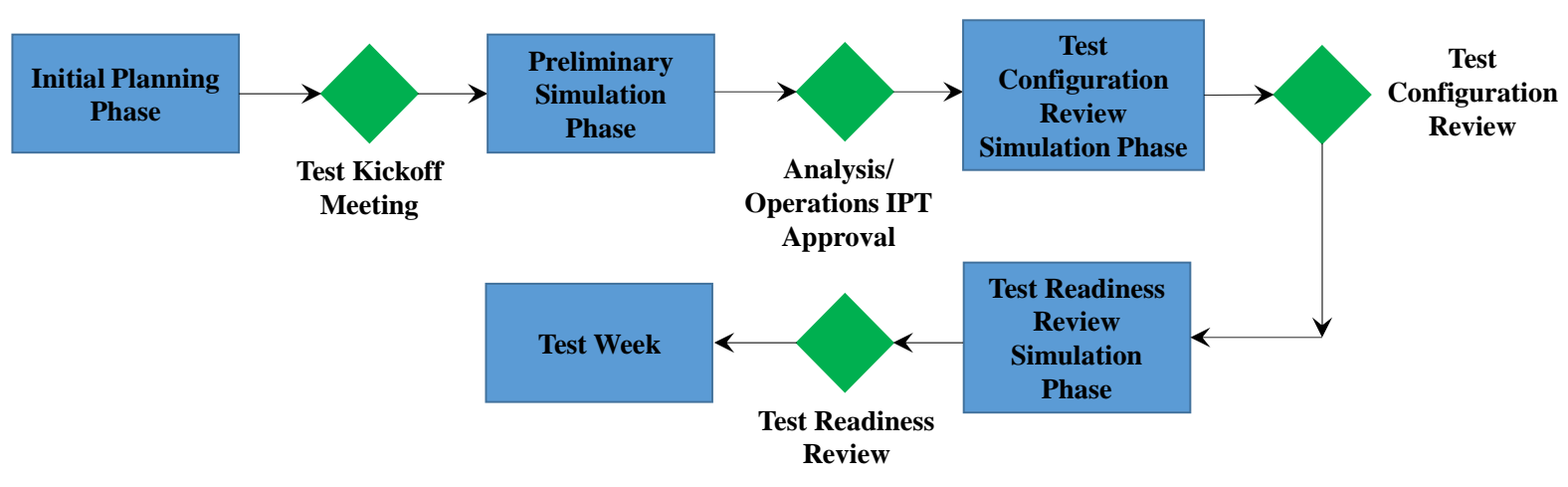

\section{Figure 1: CPAS Analysis Test Planning Process}

The process includes two trajectory simulations as well as several other tools, including a footprint tool to track released items, a load train tool to analyze loads on the extraction, programmer, and recovery parachutes, and a contact model to identify contact between parachute risers and the test vehicle. ${ }^{1}$

\section{Initial Planning Phase}

High level planning begins months, and in some cases years, in advance of a CPAS test. Refinement of the test operations concept generally occurs in Test Operations and Analysis IPT meetings where initial analyses are presented, issues and concerns are discussed, and potential test alterations can be proposed. Operational logistics of the proposed changes to the test matrix definition of the test are then discussed at a Test Operations IPT meeting and subsequently presented to CPAS Management at the Management IPT meeting for approval when necessary.

Several months before a scheduled test date, the test kickoff meeting is held. The kickoff meeting provides an opportunity for the Analysis, Operations, and Hardware teams to discuss the concept of operations and the proposed schedule for the upcoming pre-test reviews, parachute packing, and vehicle build-up. For particularly complicated tests, such as the first use of a new test vehicle or the Forward Bay Cover, the test kickoff may occur a year or more in advance of the test date, due to the unique complications of the new hardware. The kickoff meeting generally represents the point at which the test concept is sufficiently defined to allow test specific simulation development to begin. As an example, the preliminary concept of operations (conops) for CDT-3-13 is shown in Figure 2. 


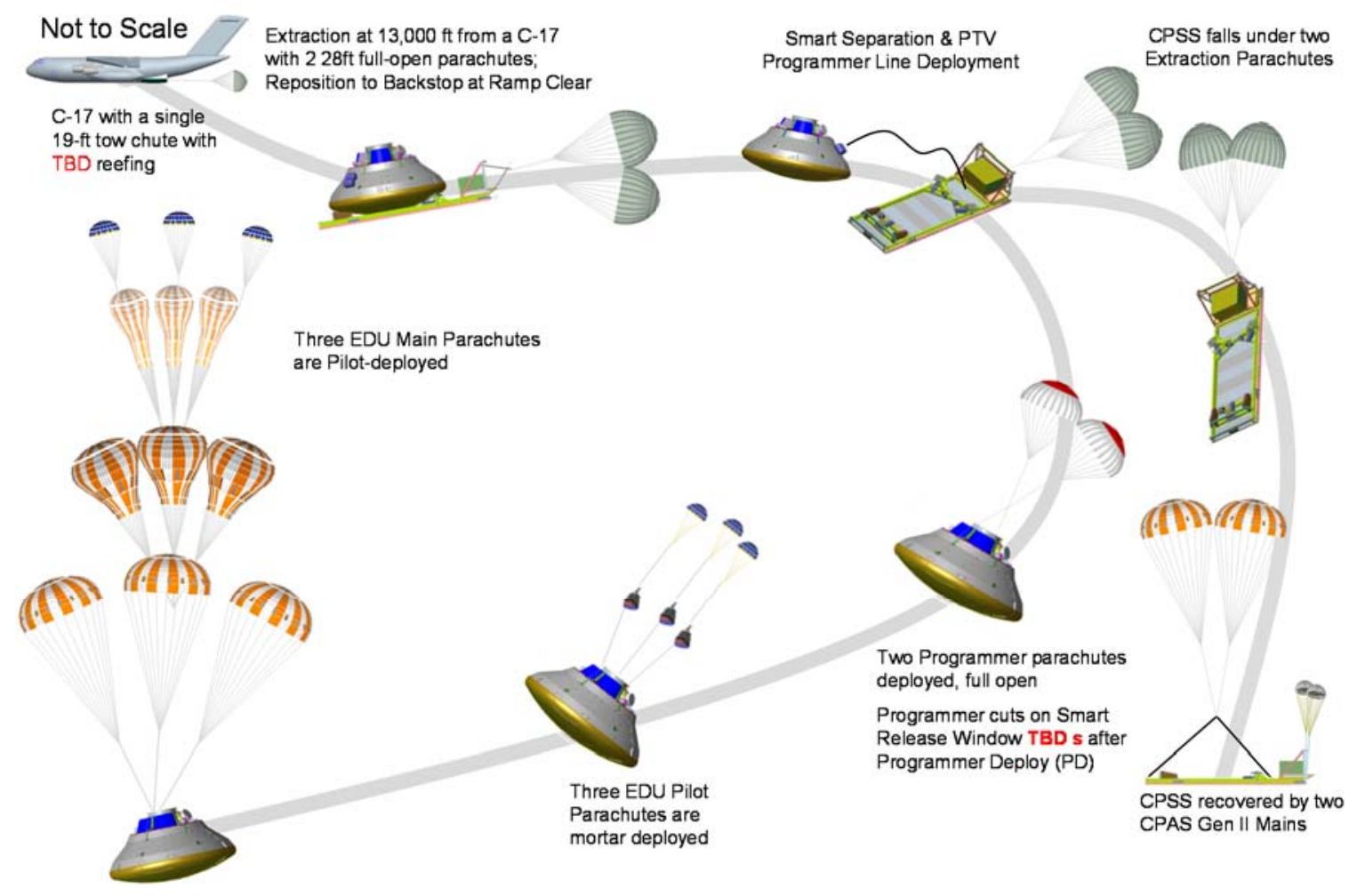

Figure 2. CDT-3-13 Preliminary Concept of Operations.

\section{Preliminary Simulation Phase}

Once the preliminary conops has been defined, the lead analyst for the drop test can begin developing the inputs for the Flight Analysis and Simulation Tool (FAST). FAST is used to model the test vehicle (e.g the Parachute Test Vehicle, or PTV) trajectory beginning after its separation from its deployment vehicle (e.g. the Cradle Platform Separation System (CPSS)) through touchdown. The Automatic Dynamic Analysis of Mechanical Systems (ADAMS) simulation is used to model the mated vehicle extraction from the aircraft and subsequent separation of the test article from its carrier platform. ${ }^{2,3}$ Since FAST requires an end state from ADAMS to define the state of the test vehicle post separation, preliminary simulation executions often use an ADAMS state from a similar previous test. This allows the PTV trajectory analyst to begin developing and refining flight trajectories, while another analyst focuses on updating ADAMS and designing the extraction/separation trajectory. Vehicle mass properties from a past test are also utilized in preliminary simulation development. Both of these steps allow the analyst to develop and communicate basic preliminary trajectory results without delay.

Once a preliminary FAST simulation is has been run, initial nominal results for one or more potential trajectories are presented to the Analysis IPT for review. These early trajectories may make numerous assumptions, including those previously mentioned, but they are reliable enough to provide a first look at viable test options. The early options are typically iterated over several weeks to refine the concept further, usually involving the performance of trade studies, after which preliminary Monte Carlo runs, typically consisting of 500 cases, may be executed to analyze the options with dispersions. Figure 3 is the result of a trade study used for CDT-3-13, analyzing the impact of two different programmer options on vehicle stability using 500-case Monte Carlo runs.

A key part of the preliminary simulation phase, particularly for unusual test objectives, is obtaining the input from the Operations and Hardware IPTs to avoid designing a trajectory that uses an exceptionally complicated or high-risk concept. An example of this is the CDT-3-13 abort straight-to-Mains test. Initially, the goal was to deploy the Main parachutes at a high capsule hang angle. The first concept was to deploy the programmer parachute to stabilize the capsule, then reposition to the desired higher hang angle. When operations concerns rendered this option infeasible, a 
second concept was studied using a second programmer to reach the desired hang angle. This was also deemed too operationally difficult. Ultimately, the high hang angle concept was discarded in favor of the low-altitude, shallow flight path angle straight-to-Mains deployment.

Once the Analysis and Operations IPTs concur on the approach for achieving the test objectives, the basic concept of operations can be considered static regarding CPAS parachutes and reefing percentages. Specific details such as event timing, programmer parachute configuration, and release altitude are still considered fluid. At this point, any atypical hardware (such as unusual reefing requirements or possible unusual programmer configurations) and avionics requirements (such as additional event sequences) have been communicated to the Operations IPT to ensure adequate time to implement the configuration.

\section{Test Configuration Review Simulation Phase}

After the test concept of operations has solidified, simulation preparation for the Test Configuration Review (TCR) begins. TCR is typically held eight to ten weeks prior to the scheduled test week and presents most aspects of the test in detail, including analysis, hardware configuration, test objectives, and avionics configuration.

During this phase, the first test-specific ADAMS extraction/separation trajectories are developed and produce initial states for the FAST simulation. Mated vehicle pitch and pitch rate limits that the avionics system uses to command the separation of the PTV from the CPSS are defined with iterative Monte Carlo ADAMS runs. The range of values defined by the pitch and pitch rate limits, combined with associated time limits, is referred to as the smart separation window ${ }^{4}$. It is designed to ensure that the PTV separates from the CPSS with pitch dynamics that prevent the PTV from entering any undesired orientation that could lead to an apex-forward attitude, tumbling, or recontact with the CPSS during programmer parachute deployment. The focus of the ADAMS analysis is to define a separation window that a high percentage of Monte Carlo cases enter and to have satisfactory vehicle stability during and after the separation event. Figure 4 shows the CDT-3-13 pitch phase plot and smart separation window, including two window options that were considered. Once the separation window is defined, the nominal and Monte Carlo ADAMS trajectory end states (at programmer deployment line stretch) are provided to the lead analyst to begin running FAST

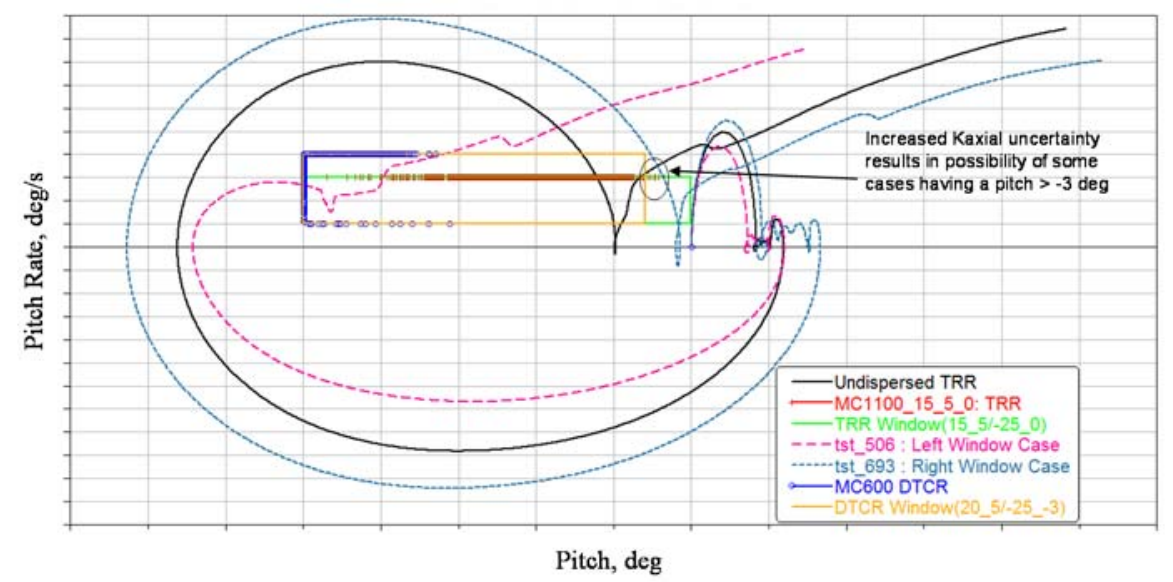

Figure 4: CDT-3-13 Pitch Phase Plot and Smart Separation Window. 
with updated trajectories. The Hardware team also provides updated mass properties for the test vehicles during this phase. These updates are still estimations of the as-built vehicle, but account for any conops changes that would cause mass changes, including number of parachutes, additional avionics, etc.

Nominal trajectory and initial Monte Carlo runs are used to refine the test configuration, particularly the programmer configuration, which is typically finalized prior to TCR. As with the nominal runs in the preliminary simulation phase, multiple scenarios are simulated to analyze some critical details. However, at this point in the process, Monte Carlo runs (typically 500-case runs) are used in scenario evaluations. For example, the CDT-3-14 simulation analysis required several iterations to determine the capsule free-fall time that maximized both the vehicle dynamic pressure at FBC deploy and the vehicle stability - an effort that continued throughout multiple phases of the test planning process.

Some tests may require modifications to FAST to add the capabilities needed to examine atypical scenarios. For CDT-3-13, FAST was modified to allow evaluation of the estimated separation distance between the PTV and CPSS at Pilot mortar fire and Main line stretch. This was a test-specific need because the straight-to-Mains test concept deployed the Main parachutes two to three seconds after PTV separation, resulting in the

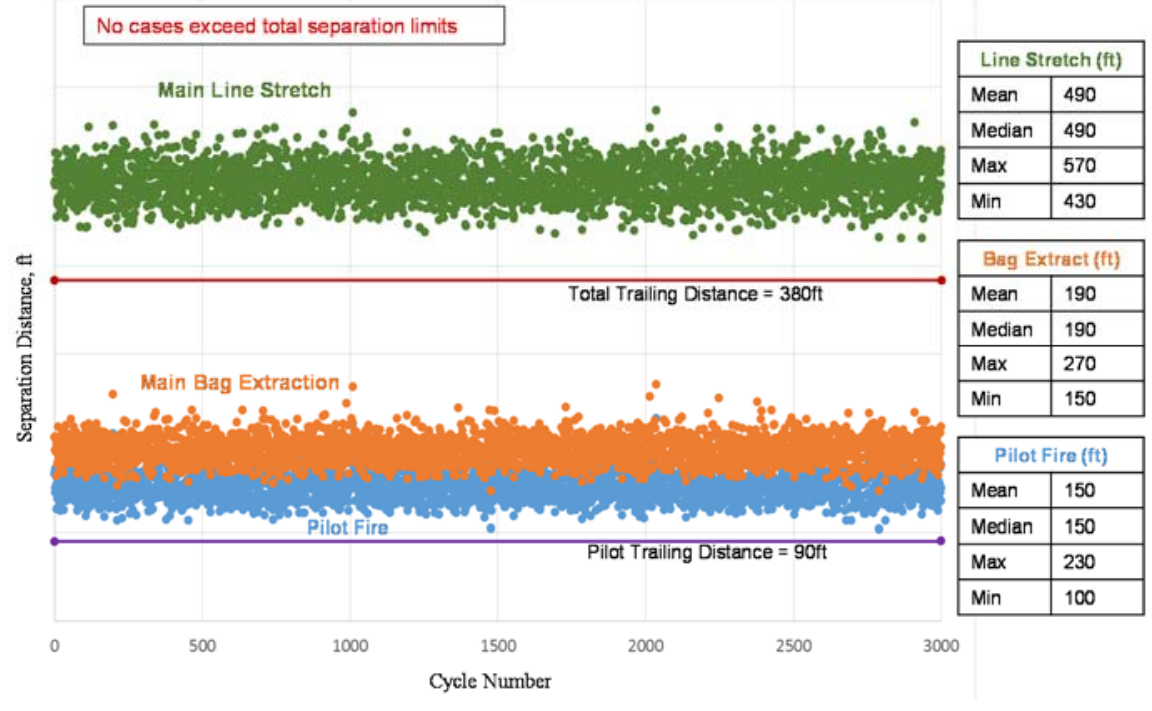

Figure 5. CDT-3-13 Monte Carlo Separation Distance Results.

PTV being in much closer proximity to the CPSS than is usually experienced at the Main deployment. Adding this capability allowed the analysts to maximize the desired shallow flight path angle while ensuring that an adequate separation distance between the two vehicles would be maintained. Examples of the nominal and Monte Carlo

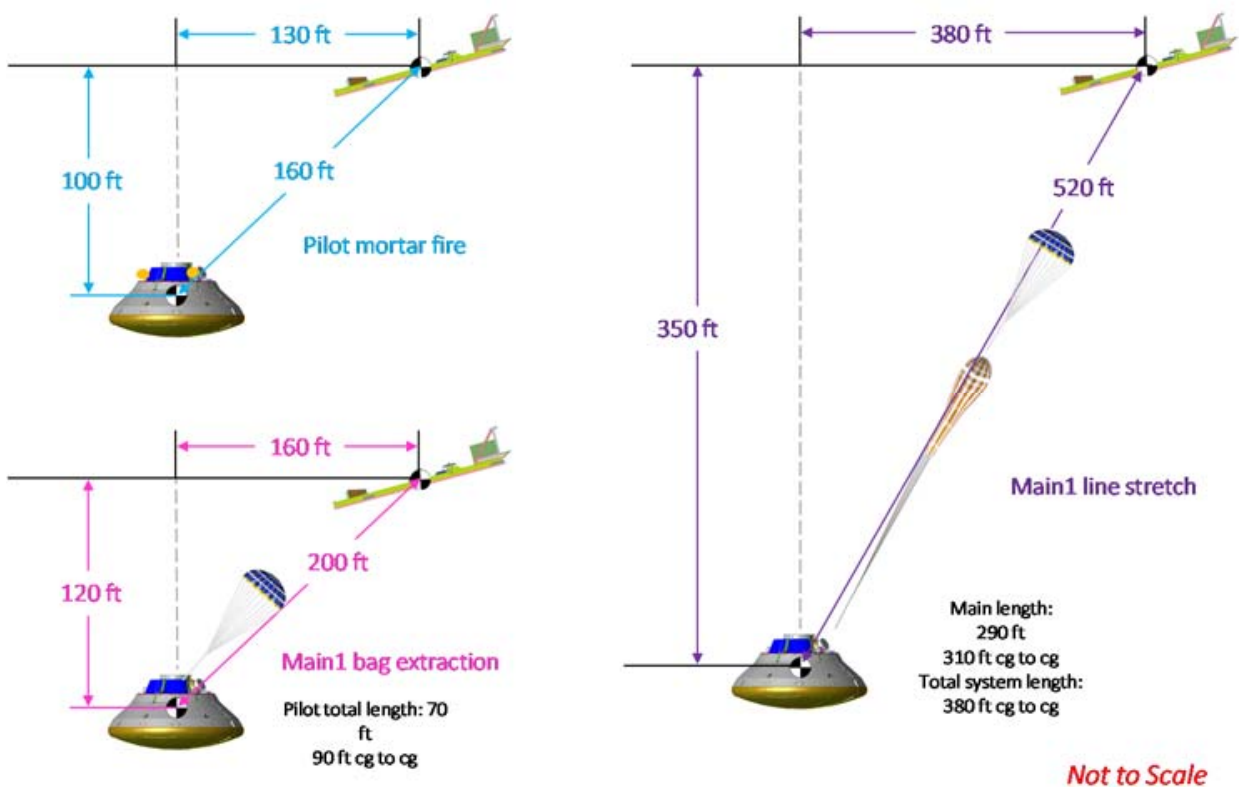

Figure 6. CDT-3-13 Nominal Separation Distance. separation distance plots are shown in Figure 5 and Figure 6.

As the preliminary Monte Carlo runs are completed, the various scenario options continue to be presented to the Analysis IPT for selection and approval, ensuring that the CPAS community, particularly IPT leads and managers, are familiar with and approves the conops prior to the TCR presentation. Once the concept is determined, a 3000- 
case Monte Carlo set is run for TCR. This Monte Carlo set is then fed into two other standalone models - a load train model and a parachute riser/PTV contact model. The load train model is used to calculate load margins for the extraction parachute system, the programmer parachutes, and the CPSS recovery parachutes, given loads and initial conditions from FAST and ADAMS. The model is also used to determine the number of energy modulators (if used) required to control the loads on the programmers and recovery parachutes. The contact model calculates the number of cases in which the Drogue, Pilot, or Main risers contact any part of the PTV based on the vehicle dynamics during flight. Highly dynamic periods during the trajectory may result in a large number of riser contacts, which increases risk of not successfully completing test objectives and could endanger the vehicle due to potential severing of the risers. In these cases, changes to the conops in order to improve vehicle stability and reduce contact risk may be made. If contact risk is a major concern, the contact model may be run on several of the 500-case scenarios prior to the TCR run to aid in choosing the optimal scenario if contact risk is a major concern. The contact model is not capable of modeling the four-point harness attach. Programmer riser and harness contacts are estimated based on the PTV total angle of attack output by FAST.

A preliminary run of the Sasquatch footprint tool ${ }^{5}$ may be done during the TCR phase to evaluate whether the released items (parachutes, mortar lids and sabots) and drop test vehicles can be expected to stay on the range given range reference mean winds for the scheduled test month. This model is not generally required until the Test Readiness Review (TRR) phase, but may be run during the TCR phase if the test concept includes high-altitude released items or vehicles spending an unusually long time under Main or recovery steady-state.

This phase concludes with the Test Configuration Review (TCR). Analysis products for TCR include a detailed summary of assumptions, analysis limitations, FAST model settings, event sequencing, ADAMS and FAST nominal and Monte Carlo results for the PTV, contact model results, and load train results. It may also include optional testspecific analyses, such as the CDT-3-13 separation distance or the CDT-3-14 freefall analysis. Preliminary nominal and Monte Carlo FAST runs for the CPSS may be available at this time or postponed until TRR. TCR is generally the point at which vehicle rigging commences, therefore all parachute details should be settled at this point.

\section{Test Readiness Review Simulation Phase}

After completion of the TCR, the Analysis team's test preparation efforts enter the TRR phase. The TRR is generally held one to two weeks prior to the scheduled test week and is the final review prior to the drop. Ideally, it follows risk review presentations to program management by at least one week, such that any changes directed by program management can be worked prior to TRR.

Changes to the conops are generally limited during this phase. Since parachute and vehicle rigging are completed prior to TRR, subsequent changes are limited to extraction release altitude and event sequencing, both of which can be altered at any point prior to test week. Depending on the amount of ongoing simulation development, there may be model updates to ADAMS and FAST, which will require new Monte Carlo runs. Updated mass properties are also delivered, reflecting measurements taken during the build-up process. The load train and contact models will be rerun once the updated 3000-case Monte Carlo sets are delivered from ADAMS and FAST. A sample plot comparing results from the CDT-3-13 Delta TCR and TRR Monte Carlo runs is shown in Figure 7.

If not completed during the TCR phase, the CPSS trajectory will be simulated in FAST

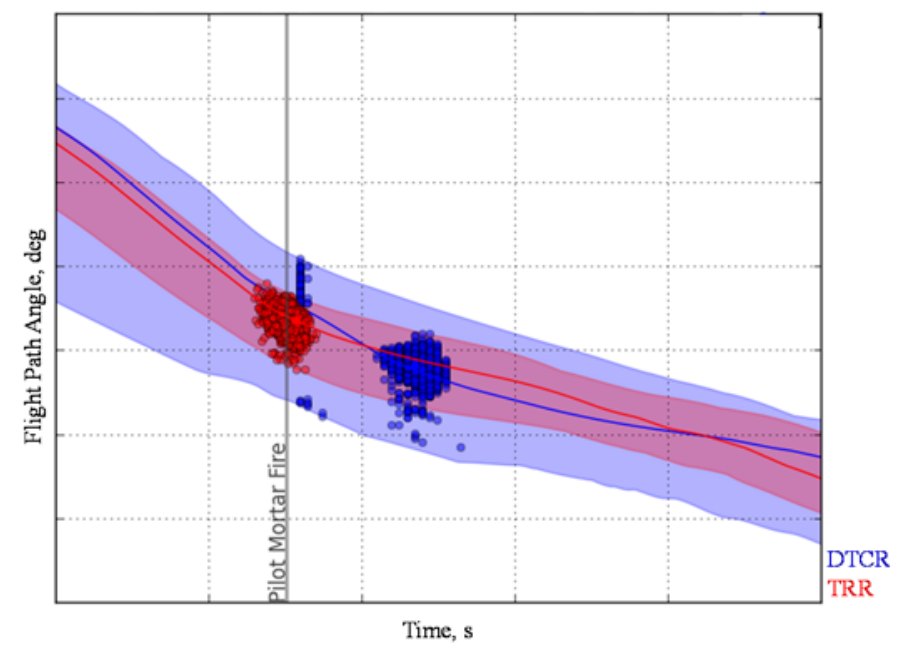

Figure 7. CDT-3-13 Delta TCR vs TRR Monte Carlo Results. during this phase. A separate 3000-case Monte Carlo is used for this vehicle.

The Sasquatch footprint simulation will also be updated with any new test-specific released items and run using the YPG range reference mean winds for the month of the drop test. Depending on the results of the Sasquatch run, a wind contingency case may be developed. This provides an option to lower the release altitude on the day of the test without altering any other part of the trajectory in order to control the footprint of the vehicle and released items. Not 
all tests have a wind contingency option, since the lowered release altitude removes steady-state altitude at the terminal phase of the descent; the Main and recovery parachutes must reach steady-state at a sufficiently high altitude to achieve test objectives and to ensure that the vehicle has time to slow enough to land safely.

The phase concludes with the Test Readiness Review (TRR). The Analysis Team's inputs are largely the same as the TCR inputs, changes made to the models and trajectories during the TRR phase are highlighted.

\section{Test Week}

The Analysis Team's role during test week involves real-time test support and execution of the Sasquatch footprint tool at the drop test site. Two team members support the test as lead and backup analysts. Beginning at the end of the week prior to the test, a Sasquatch analyst will run the tool using the forecasted winds for test day. Additional runs will be executed as the forecast is updated in the days leading up to the test. On the day of the test, weather balloons are released at one-hour intervals beginning several hours prior to the scheduled time of test and continuing until after the test is complete. The balloons provide atmospheric data, including winds aloft, that the analyst inputs into Sasquatch. Sasquatch outputs are used to select the point at which the aircraft will target the test vehicle release in order to ensure that the vehicle and released objects land within range limits. If the winds are particularly high and the footprint violates range limits, the wind contingency case (if available) may be implemented, lowering the aircraft release altitude to decrease the amount of time the released objects are in the air. Figure 8 is an example of the footprint completed for the CDT-3-13 TRR.

Sasquatch also outputs files containing predicted position traces of all released items with respect to time. These files are input into the Advanced Cockpit Environment Simulation (ACES), a program used by the chase helicopters observing the test in order to assist the helicopters with debris avoidance. The release point is generally finalized and the ACES file delivery completed approximately two hours prior to the start of the test. The Sasquatch analysts continue

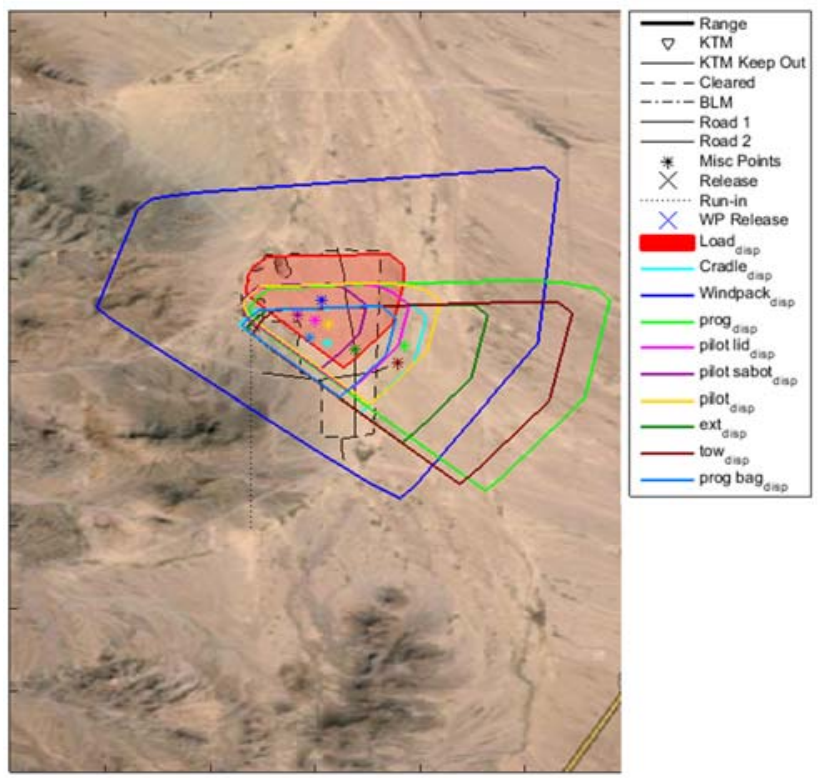

Figure 8. CDT-3-13 Sasquatch Footprint Prediction. analyzing the footprint with each new set of atmospheric data, but will not alter the release point unless a significant wind shift changes the footprint. If a new release point must be chosen after the aircraft is in the air, it will be communicated to the aircraft by range personnel. A Sasquatch analyst will also provide predicted touchdown locations for all released items to the recovery team, in order to expedite the search for the released items. After the test is complete, the analysts participate in a review of the available test videos and still photos at the test facility, attempting to identify any anomalous or unexpected behavior involving the parachutes or vehicles.

\section{Conclusion}

The CPAS Analysis Team process for drop test preparation has been refined over the eight years of testing that has been conducted to date, and will continue to be improved over the remainder of the life of the test program. Fifteen drop tests have been conducted since the start of the EDU test program in 2011, including Forward Bay Cover tests, abort scenarios, and simulated parachute failures, with two EDU tests remaining prior to the Critical Design Review and qualification testing. The Analysis Team has relied heavily on communication and team flexibility in order to meet an aggressive test schedule. This process, and the level of communication it encourages, is a key component of the Analysis Team's ability to simultaneously prepare for several drop tests, improve parachute simulations, and react quickly to new information and evolving test requirements. 


\section{References}

${ }^{1}$ Romero, L.M., et al., "Summary of CPAS EDU Testing Analysis Results," 23 ${ }^{\text {rd }}$ AIAA Aerodynamics Decelerator Systems Technology Conference, Daytona, Florida, March 2015 (submitted for publication).

${ }^{2}$ Fraire, U., Anderson, K., and Cuthbert, P.A., "Extraction and Separation Modeling of Orion Test Vehicles with ADAMS Simulation,” 22 ${ }^{\text {nd }}$ AIAA Aerodynamics Decelerator Systems Technology Conference, Daytona, Florida, March 2013, AIAA paper 2013-1394.

${ }^{3}$ Fraire, U. and Varela, J.G., “ADAMS Model Improvements (TBD title)," $23^{\text {rd }}$ AIAA Aerodynamics Decelerator Systems Technology Conference, Daytona, Florida, March 2015 (submitted for publication).

${ }^{4}$ Moore, J.W. and Morris, A.L., "Development of a Smart Release Algorithm for Mid-Air Separation of Parachute Test Articles," $21^{\text {st }}$ AIAA Aerodynamics Decelerator Systems Technology Conference, Dublin, Ireland, May 2011, AIAA paper 2011-2602.

${ }^{5}$ Bledsoe, K.J. and Bernatovich, M.A., "Development and Overview of CPAS Sasquatch Airdrop Landing Location Predictor Software,” $23^{\text {rd }}$ AIAA Aerodynamics Decelerator Systems Technology Conference, Daytona, Florida, March 2015 (submitted for publication). 